\title{
Intoxication massive au lithium associée à une alopécie télogène diffuse
}

\author{
Acute lithium intoxication with diffuse \\ telogen effluvium
}

\section{Elodie SAUSSEREAU ${ }^{(1) *}$, Christian LACROIX ${ }^{(1)}$, Pierre BRAVARD $^{(2)}$, Jean NOUVEAU ${ }^{(3)}$, Jean- Pierre GOULLE ${ }^{(1)}$}

(1) Laboratoire de Pharmacocinétique et de Toxicologie Cliniques (2) Service de Dermatologie (3) Service de Réanimation Médicale. Groupe Hospitalier du Havre, BP 24, 76083 LE HAVRE.

*Auteur à qui adresser la correspondance : Élodie SAUSSEREAU

Laboratoire de Pharmacocinétique et de Toxicologie Cliniques, Groupe Hospitalier du Havre, BP 24, 76083 LE HAVRE - Tél : 0232733218 - Fax : 0232733238 -E-mail : esaussereau@ch-havre.fr

(Reçu le 12 octobre 2006 ; accepté après modifications le 31 janvier 2007)

\section{$\boldsymbol{R E} \boldsymbol{E} U \boldsymbol{M} \boldsymbol{E}$}

L'observation rapportée concerne un cas d'intoxication massive au lithium chez une femme de 38 ans, ayant ingéré jusqu'à 120 comprimés de Teralithe LP® $400 \mathrm{mg}$. Cette patiente, présentant des troubles de l'humeur bipolaires, avait arrêté son traitement au lithium un mois avant l'intoxication. La malade a présenté des troubles de la vigilance ainsi qu'une défaillance multi-viscérale et hémodynamique. Au $15^{\text {ème }}$ jour de l'intoxication, à l'extubation, une zone d'alopécie occipitale avec atteinte diffuse du cuir chevelu a été observée. L'évolution des concentrations du lithium a été étudiée à partir de prélèvements sanguins. Les dosages plasmatiques et érythrocytaires ont été effectués par photométrie de flamme. Un prélèvement capillaire, effectué au 30 ème jour de l'intoxication, a permis l'étude des cheveux en microscopie optique et le dosage du lithium avec une torche à plasma couplée à un spectromètre de masse. Quarante huit heures après l'ingestion du lithium sous forme à libération prolongée, les concentrations plasmatiques et intra-érythrocytaires étaient respectivement de 14,90 et 12,10 mmol/L. L'épuration du lithium a été effectuée par

\section{SUMMARY}

In the presented case, a 38 year old woman had taken until 120 tablets of Teralithe LP® $400 \mathrm{mg}$. This woman who presented bipolar affective disorders had stopped her treatment with lithium one month before the intoxication. The patient was initially confused, and developed afterwards general and hemodynamic seizures. Fifteen days later, at the time of extubation, a diffuse effluvium was observed in the occipital region. The lithium pharmacokinetic was studied in blood samples. Quantitative determination in plasma and erythrocytes was done by flame photometry. Hair samples obtained from occipital site one month after the intoxication, were observed in optic microscopy and the lithium concentration was measured by ICP-MS. Forty eight hours after the massive ingestion of sustained-release lithium preparation, plasmatic and intraerythrocytic levels were respectively 14,90 and $12,10 \mathrm{mmol} / \mathrm{L}$. Continuous venovenous haemodialysis was performed for 3 days. Plasmatic and intraerythrocytic concentrations were below the limit of detection after 8 days. The hair lithium concentrations were respectively in roots and tips, 
hémodiafiltration veino-veineuse continue pendant 3 jours. L'obtention de concentrations plasmatiques et globulaires inférieures à 0,05 mmol/L a nécessité 8 jours. Dans les cheveux, la teneur en lithium était respectivement au niveau des racines et des pointes, de 1,83 et 2,17 $\mathrm{ng} / \mathrm{mg}$. L'examen en microscopie optique a mis en évidence $60 \%$ de cheveux télogènes. La malade, précédemment traitée avec du Teralithe LP ${ }^{\circledR} 400 \mathrm{mg}$, a survécu à cette intoxication massive malgré des concentrations plasmatiques et intra-érythrocytaires considérables, jamais rapportées chez un sujet vivant.

\section{MOTS-CLÉS}

Lithium, intoxication aiguë, cheveux, télogène.

\section{Introduction}

Le lithium, métal alcalin, est le chef de file des thymorégulateurs ou normothymiques. Il a un effet préventif sur les troubles récidivants de l'humeur des psychoses maniaco-dépressives et un effet curatif dans les accès maniaques et les accès dépressifs. L'index thérapeutique du lithium est étroit, les concentrations thérapeutiques plasmatiques étant comprises entre 0,60 à $1,20 \mathrm{mmol} / \mathrm{L}$ (jusqu'à $1,50 \mathrm{mmol} / \mathrm{L}$ dans les accès maniaques) (1). La forme active du lithium étant la forme intracellulaire, des effets indésirables peuvent être observés à des concentrations proches des valeurs normales, d'où la nécessité d'effectuer régulièrement des dosages plasmatiques et intra-érythrocytaires chez les malades traités afin d'adapter la posologie. Le lithium est totalement absorbé au niveau de la partie supérieure du tractus gastro-intestinal en 8 heures, avec un pic plasmatique 1 à 2 heures après l'administration per os (1). Ce pic plasmatique est retardé en cas d'administration d'une forme à libération prolongée. D'autre part, en cas d'intoxication, l'absorption du lithium est également prolongée du fait de la formation d'agrégats de sels de lithium plus ou moins solubles ; ce phénomène est très important avec les formes à libération prolongée (2). La distribution tissulaire du lithium est relativement complexe après son ingestion, avec un décalage de 24 heures au niveau du tissu cérébral par rapport au plasma (1). Le lithium n'est pas métabolisé dans l'organisme et son élimination est rénale. La demivie d'élimination dans le sang varie avec l'âge, la durée d'exposition au lithium et l'état de la fonction rénale. Elle est de l'ordre de 22 heures mais peut atteindre 58 heures dans le cas d'un traitement chronique (1). Il existe trois types d'intoxication au lithium : intoxication aiguë chez un sujet non traité, intoxication aiguë chez un patient sous traitement, surdosage chronique. En cas d'intoxication chez un sujet non traité par du lithium, il existe une forte discordance entre les concentrations plasmatiques parfois très élevées $(>5 \mathrm{mmol} / \mathrm{L})$ et la clinique discrète : somnolence et/ou diarrhées. En
1,83 and $2,17 \mathrm{ng} / \mathrm{mg}$. The trichogram revealed $60 \%$ of telogen hairs. This patient, treated with Teralithe LP® $400 \mathrm{mg}$, has lived through this acute intoxication despite very high plasmatic and intraerythrocytic levels of lithium, never described in medical literature in a survived patient.

\section{KEY-WORDS}

Lithium, overdose, hair, telogen.

revanche, une intoxication aiguë chez un patient sous traitement avec du lithium est généralement associée à une symptomatologie clinique sévère (encéphalopathie, crises convulsives). La demi-vie d'élimination dans ce dernier cas est plus longue que chez un sujet naïf et des concentrations plasmatiques de l'ordre de 3 à $4 \mathrm{mmol} / \mathrm{L}$ nécessitent une hémodialyse, en particulier si la fonction rénale est altérée $(1,3)$. Dans le cas d'une intoxication avec une forme à libération prolongée, l'hémodiafiltration veino-veineuse continue est plus efficace en raison du risque d'accumulation notamment intracellulaire, et limite le phénomène de relargage (1).

\section{Observation}

Une femme âgée de 38 ans, ayant ingéré au cours d'une tentative d'autolyse jusqu'à 120 comprimés de Teralithe $\mathrm{LP}{ }^{\circledR} 400 \mathrm{mg}$, est hospitalisée pour une intoxication aiguë et sévère au lithium. Cette patiente, suivie en psychiatrie pour des troubles de l'humeur bipolaires, avait arrêté son traitement au lithium (Teralithe LP® $400 \mathrm{mg}$, deux comprimés par jour) un mois avant cette ingestion massive.

Quelques heures après l'intoxication, la patiente est admise en réanimation médicale et un lavage gastrique est réalisé ainsi qu'une réhydratation avec du sérum physiologique. La lithiémie plasmatique est alors de $2,50 \mathrm{mmol} / \mathrm{L}$. La patiente fortement agitée est transférée en psychiatrie. Quarante huit heures après l'ingestion massive, la malade présentant des troubles de la vigilance est réadmise aux urgences. Le bilan biologique met en évidence une lithiémie plasmatique très élevée, $14,90 \mathrm{mmol} / \mathrm{L}$, associée à une insuffisance rénale aiguë (créatinine plasmatique : $409 \mu \mathrm{mol} / \mathrm{L}$ ). Une défaillance multi-viscérale est rapidement constatée, avec une défaillance respiratoire nécessitant une intubation orotrachéale, ainsi qu'une défaillance hémodynamique sans réponse initiale au remplissage, ni aux amines vasopressives. Une hémodialyse est mise en place du fait d'une insuffisance rénale anurique. L'épuration 
du lithium est réalisée par hémodiafiltration veinoveineuse continue pendant 3 jours. L'évolution sera progressivement favorable sur le plan hémodynamique

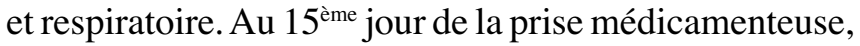
à l'extubation, une zone d'alopécie occipitale au niveau de la zone d'appui sur l'oreiller est observée. La disparition des cheveux est complète, donnant un aspect peladique avec peau blanche sans follicule pileux visible (Figure 1). L'atteinte diffuse est confirmée par un test de traction positif en tout point du cuir chevelu. Ce test permet, de manière simple, par la traction d'une dizaine de cheveux pincés entre le pouce et l'index en différents points du cuir chevelu, d'apprécier l'importance d'une chute de cheveux (normalement limitée à 1 à 2 cheveux après traction de 10 cheveux). Des prélèvements de cheveux sont effectués au $30^{\text {ème }}$ jour de l'intoxication, pour l'étude en microscopie optique et le dosage du lithium. L'alopécie diffuse du cuir chevelu est réversible ; des cheveux de repousse sont observés au niveau de la zone alopécique malgré la persistance de l'effluvium.

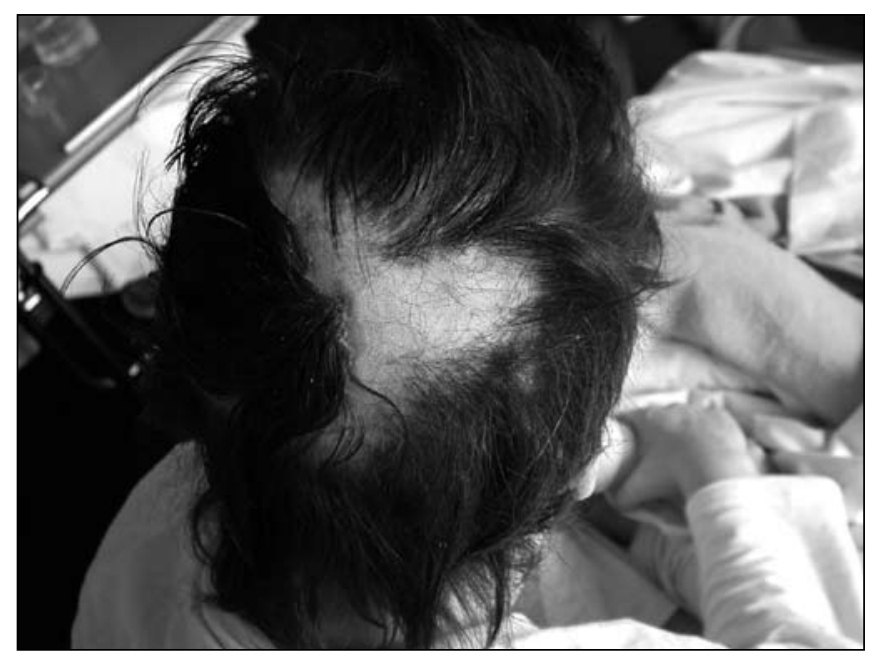

Figure 1 : Alopécie diffuse occipitale au niveau de la zone d'appui sur l'oreiller.

\section{Matériel et méthodes}

\section{Lithium plasmatique et érythrocytaire}

Le dosage est réalisé par spectrophotométrie d'émission de flamme.

\section{Réactifs}

L'ensemble des réactifs est au minimum de qualité analytique : eau purifiée Aqua B. Braun, Melsungen, Allemagne (Réf. 0066571E), étalon de lithium à $1 \mathrm{~g} / \mathrm{L}$ Fluka, Buchs, Suisse (Réf. 62372), chlorure de sodium à $0,9 \%$ Aqua B. Braun, Melsungen, Allemagne (Réf. 0066570E).

\section{Préparation de l'échantillon}

Le plasma est dilué au 1/100 dans l'eau purifiée. Les étalons $(0,36-0,72-1,44 \mathrm{mmol} / \mathrm{L})$ sont traités dans les mêmes conditions. Les érythrocytes sont lavés avec du chlorure de sodium à $0,9 \%$; après centrifugation, le surnageant est éliminé. Après une dilution au 1/50 dans l'eau purifiée, les érythrocytes sont hémolysés à $4^{\circ} \mathrm{C}$ pendant 15 minutes. Après centrifugation, le dosage est effectué sur le surnageant.

\section{Instrumentation}

Les analyses ont été réalisées à l'aide d'un spectromètre d'absorption atomique VARIAN AA 1475 utilisé source éteinte, équipé d'un passeur d'échantillon programmable VARIAN PSC 55. La longueur d'onde est réglée à $670,8 \mathrm{~nm}$; la fente à $0,5 \mathrm{~nm}$. Le réglage de la flamme est optimisé avec une solution de lithium à $0,05 \mathrm{mmol} / \mathrm{L}$. L'appareil est relié à une imprimante Epson RX 80.

\section{Lithium dans les cheveux}

\section{Réactifs}

Les réactifs, de qualité suprapure pour analyse de traces et la solution étalon à $10 \mathrm{mg} / \mathrm{L}$ (ICP Multi Element Standard Solution VI CertiPUR - Réf. 1.10580.0100) proviennent de chez Merck (Darmstadt, Allemagne) et CPI (Amsterdam, Hollande).

\section{Préparation de l'échantillon}

Le dosage du lithium a été réalisé dans une mèche de cheveux de $5 \mathrm{~cm}$ de long prélevée au niveau de la racine et segmentée en 5 fragments de $1 \mathrm{~cm}$. Après décontamination par de l'acétone et de l'eau tiède, $25 \mathrm{mg}$ de cheveux sont minéralisés par $250 \mu \mathrm{L}$ d'acide nitrique suprapur à $70^{\circ} \mathrm{C}$ pendant une heure (soit $10 \mathrm{mg}$ pour $100 \mu \mathrm{L}$ ). La solution est ensuite refroidie une heure à $-20^{\circ} \mathrm{C}$. A $100 \mu \mathrm{L}$ de la solution acide obtenue, sont ajoutés $3900 \mu \mathrm{L}$ de diluant $(0,5 \%$ de butanol, $1 \%$ d'acide nitrique, $0,01 \%$ de triton, Indium et Rhodium, étalons internes, à une concentration de $1 \mu \mathrm{g} / \mathrm{L}$ ). L'étalonnage est réalisé dans de l'eau surchargée à 0,5 - 1,0 - 3,0 6,0 - 12,0 - 24,0 $\mu \mathrm{g}$ de lithium par litre.

\section{Instrumentation}

Les analyses ont été réalisées à l'aide d'une torche à plasma de type ThermoElectron X Series couplée à un spectromètre de masse, modèle X7/CCT (ThermoElectron, Courtabœuf, France). L'appareil est équipé d'une torche en quartz de $1,5 \mathrm{~mm}$, d'un nébuliseur concentrique en verre borosilicaté de $1 \mathrm{~mL}$ (type concentrique de marque Glass Expansion - Référence ThermoElemental 1201318) avec un débit d'échantillon de $0,85 \mathrm{~mL} / \mathrm{min}$, d'une chambre de nébulisation en quartz munie d'un refroidisseur à effet Peltier régulant sa température à $3^{\circ} \mathrm{C}$, et d'un passeur d'échantillon de type CETAC ASX-510. L'ensemble des données est enregistré sur une station informatique dotée du logiciel d'analyse PlasmaLab version 2.0 sous Windows NT. Le dosage s'effectue selon des paramètres instrumentaux déjà décrits (4). 


\section{Résultats}

La concentration plasmatique du lithium, dans le premier prélèvement réalisé dans les heures suivant l'ingestion massive du Teralithe ${ }^{\circledR}$ LP, était de $2,50 \mathrm{mmol} / \mathrm{L}$. A 48 heures de l'intoxication, la concentration plasmatique du lithium était de $14,90 \mathrm{mmol} / \mathrm{L}$ et la concentration érythrocytaire, reflet de la forme active intracellulaire et des phénomènes centraux d'accumulation, de $12,10 \mathrm{mmol} / \mathrm{L}$, soit un rapport érythro-plasmatique de 0,87 . Une décroissance rapide des lithiémies plasmatiques et érythrocytaires a été observée au cours des 24 heures suivantes ( $\mathrm{T} 48$ à $\mathrm{J}$ 3) (Figure 2). L'obtention de concentrations plasmatiques et globulaires inférieures ou égales à $0,05 \mathrm{mmol} / \mathrm{L}$ a nécessité un peu plus de 8 jours.

Dans les cheveux, les teneurs en lithium déterminées

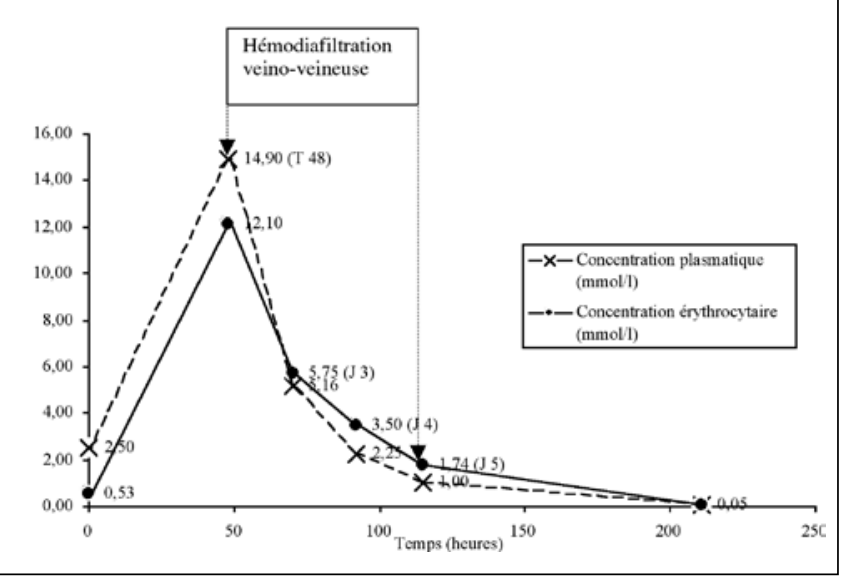

Figure 2 : Toxicocinétique du lithium dans le plasma et les érythrocytes.

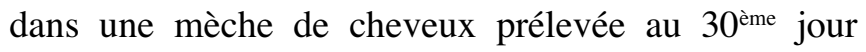
de l'intoxication, étaient de $1,83 \mathrm{ng} / \mathrm{mg}$ au niveau des racines et de $2,17 \mathrm{ng} / \mathrm{mg}$ au niveau des pointes (Figure 3). Leur examen au microscope optique a mis en évidence $60 \%$ de cheveux en phase télogène, $10 \%$ en phase anagène et $5 \%$ de cheveux dysplasiques.

\begin{tabular}{|c|c|c|c|c|c|}
\hline \multicolumn{2}{|c|}{ Pointe } & & & & Racine \\
\hline \multirow{3}{*}{$\frac{\text { Lithium }}{(\mathrm{ng} / \mathrm{mg})}$} & $\downarrow$ Sept-05 & Oct-05 & Nov-05 & Déc-05 & Janv-06 $\downarrow$ \\
\hline & $\longrightarrow 2.17$ & 1.95 & 1,99 & 2.61 & 1.83 \\
\hline & Téralit & P $400 \mathrm{mg}$ & & the $e^{\odot}$ & $\begin{array}{l}\text { Téralithe }{ }^{\oplus} \text { LP } 400 \mathrm{mg} \\
\underline{120 \mathrm{cps}}\end{array}$ \\
\hline
\end{tabular}

Figure 3 : Concentration en lithium dans une mèche de cheveux de $5 \mathrm{~cm}$ prélevée à $J+30$.

\section{Discussion}

Cette observation met en évidence les problèmes spécifiques de l'intoxication au lithium sous forme à libération prolongée. En effet, dans le cas d'une ingestion massive, l'absorption du lithium est prolongée en raison de la formation d'agrégats plus ou moins insolubles de sels de lithium (2). Ce phénomène est d'autant plus important que le lithium ingéré est sous forme à libération prolongée, et est à l'origine d'un pic plasmatique tardif pouvant persister plusieurs heures. Le risque principal en cas d'intoxication avec une forme à libération prolongée est l'absence de signes cliniques ou une symptomatologie très discrète plusieurs heures après l'ingestion. Dans le cas décrit, la défaillance multi-viscérale a été observée à partir de la $48^{\text {ème }}$ heure de l'intoxication. Le délai existant entre l'ingestion et l'apparition des signes cliniques impose, en cas de suspicion d'intoxication au lithium sous forme à libération prolongée, de réaliser au minimum deux dosages à quelques heures d'intervalle afin de s'assurer d'une décroissance des concentrations.

L'alopécie diffuse observée au niveau occipital est probablement due à la toxicité directe du lithium sur les follicules pileux. En effet, l'étude au microscope optique a mis en évidence $60 \%$ de cheveux en phase télogène associée à des follicules au repos. Cette phase télogène est caractérisée par une forte activité de l'adénylate cyclase et une concentration élevée d'AMP cyclique ayant pour conséquence une inhibition des mitoses en phase G1 (5). Or, le lithium induirait l'activité de base de l'adénylate cyclase dans certains tissus (6). D'autre part, le lithium inhiberait la glycogène synthase kinase-3 (GSK-3), enzyme intervenant dans la morphogenèse des follicules pileux et dans la différenciation et la prolifération des follicules matures $(7,8)$. Cette alopécie télogène est vraisemblablement à l'origine de l'absence d'incorporation significative du lithium dans les cheveux malgré l'ingestion massive. Nous n'avons pas noté dans cette observation, de contamination des segments analysés par la sueur ou le sébum. L'augmentation relative de la teneur en lithium du $4^{\text {ème }}$ fragment de la mèche de cheveux $(2,61 \mathrm{ng} / \mathrm{mg}$; figure 3$)$ pourrait être due à une prise médicamenteuse occulte, non contrôlée médicalement. Une autre hypothèse serait un chevauchement des 4 ème et $5^{\text {ème }}$ fragments, l'intoxication se situant à la jonction de ces deux fragments. Il aurait pu être intéressant de segmenter davantage ces deux fragments afin de repérer un éventuel impact de l'ingestion massive du Teralithe ${ }^{\circledR}$ sur la teneur en lithium des cheveux de la patiente.

\section{Conclusion}

La malade, précédemment traitée avec du Teralithe $\mathrm{LP} \otimes 400 \mathrm{mg}$, a survécu à cette intoxication massive au lithium malgré des concentrations plasmatiques et intraérythrocytaires tout à fait considérables, respectivement 
14,90 et $12,10 \mathrm{mmol} / \mathrm{L}$. Il s'agit des concentrations les plus élevées jamais rapportées chez un sujet non décédé. En effet, des concentrations plasmatiques supérieures à $4 \mathrm{mmol} / \mathrm{L}$ sont considérées comme pouvant avoir une conséquence létale chez un sujet traité ou ayant arrêté son traitement au lithium peu de temps avant l'intoxication aiguë. L'atteinte télogène diffuse du cuir chevelu observée chez la patiente est probablement la conséquence directe de la toxicité du lithium sur les follicules pileux, pouvant expliquer les faibles concentrations de lithium mises en évidence dans les cheveux prélevés.

\section{Références}

1. Timmer R.T., Sands J.M. Lithium intoxication. J. Am. Soc. Nephrol. 1999 ; 10 : 666-74.

2. Astruc B., Petit P., Abbar M. Overdose with sustainedrelease lithium preparations. Eur. Psychiatry 1999 ; 14 : 172-74.

3. Goldfrank L.R., Osborn H., Weisman R.S. Lithium. In : 4th ed. Gold-frank's toxicology emergency. Norwalk, Connecticut : Appleton and Lange, 1990 ; 427-31.

4. Goullé J.P., Mahieu L., Bonneau L., Lainé G., Bouige D., Lacroix C. Validation d'une technique de dosage multiélémentaire des métaux et métalloïdes dans les cheveux par ICP-MS. Valeurs de référence chez 45 témoins. Ann. Toxicol. Anal. $2005 ; 17$ : 97-103.

5. Cipriani C., Moretti G., Rampini E., Divano C. AdenylCyclase Activity in Rat-Hair-Cycle. Arch. Derm. Res. $1976 ; 256: 319-25$.

6. Quiroz J.A., Gould T.D., Manji H.K. Molecular effects of lithium. Mol. Interv. 2004 ; 4 : 259-72.

7. Oshima H., Rochat A., Kedzia C., Kobayashi K., Barrandon Y. Morphogenesis and renewal of hair follicles from adult multipotent stem cells. Cell. 2001 ; 104 : 233-45.

8. McCormick J.A, Feng Y., Dawson K., Behne M.J., Yu B., Wang J., Wyatt A.W., Henke G., Grahammer F., Mauro T.M., Lang F., Pearce D. Targeted disruption of the Protein Kinase SGK3/CISK impairs postnatal hair follicle development. Mol. Biol. Cell. 2004 ; 15 : 4278-88. 